\title{
Dexmedetomidine suppresses the development of abdominal aortic aneurysm by downregulating the mircoRNA-21/PDCD 4 axis
}

\author{
QI YU ${ }^{1}$, QIANQIAN LI ${ }^{1}$, XINGLONG YANG ${ }^{2}$, QIANG LIU $^{2}, J_{U N}$ DENG $^{2}$, \\ YANPING ZHAO ${ }^{2}$, RUILIN HU ${ }^{2}$ and MIN DAI $^{2}$ \\ Departments of ${ }^{1}$ Anesthesiology and ${ }^{2}$ General Surgery, The First Affiliated Hospital of Nanchang University, \\ Nanchang, Jiangxi 330006, P.R. China
}

Received January 3, 2020; Accepted January 27, 2021

DOI: $10.3892 /$ ijmm.2021.4923

\begin{abstract}
Abdominal aortic aneurysm (AAA) is a pathological state with permanent dilation, which indicates a fatal potential for aortic rupture. It has been reported that dexmedetomidine (Dex) and microRNA (miR)-21 are involved in the progression of AAA. Thus, the present study aimed to investigate the joint effects of these factors on AAA treatment. For this purpose, rat models of AAA were established with enzyme perfusion and the rats were then injected with Dex. Alterations in the abdominal aorta in rats with AAA were recorded. miR-21 expression in the rats with AAA was determined. Inflammatory factor expression was detected by western blot analysis. Subsequently, a dual-luciferase reporter gene assay was performed to verify the targeting association between miR-21 and programmed cell death protein 4 (PDCD4). Additionally, AAA-related indices and inflammatory responses were examined by an injection of a combination of antagomiR (ant)-miR-21 and Dex or lentivirus-PDCD4-short hairpin RNA. It was found that Dex markedly alleviated the development of AAA and downregulated the expression of inflammatory factors and matrix metalloproteinase in rats with AAA. The high expression of miR-21, which targets PDCD4, was observed in the rats with AAA. However, ant-miR-21 induced AAA development and inflammatory responses. Additionally, the inhibition of PDCD4 reduced AAA development and inflammatory responses. On the whole, the present study demonstrates that Dex inhibits AAA development by downregulating the miR-21/PCDP4 axis. The findings of the present study may provide novel insight for the treatment of AAA. These findings may provide a reference for the future treatment of AAA and may provide theoretical guidance for the early prevention and development of AAA.
\end{abstract}

Correspondence to: Dr Min Dai, Department of General Surgery, The First Affiliated Hospital of Nanchang University, 17 Yongwaizheng Street, Nanchang, Jiangxi 330006, P.R. China E-mail: drdaimin0906@163.com

Key words: dexmedetomidine, abdominal aortic aneurysm, microRNA-21, PDCD4, NF- $\mathrm{B}$

\section{Introduction}

Abdominal aortic aneurysm (AAA) represents a fatal human vascular disease and a chronic degenerative pathological process in which inflammatory factors and activated matrix metalloproteinases (MMPs) are highly expressed (1). AAA can be triggered by a family history of AAA, hypertension, coronary artery disease, myocardial infarction and peripheral artery injury, and males $>65$ years with a history of smoking are the most vulnerable at-risk population for the development of AAA (2). As a public health concern of utmost importance and severity, AAA has an incidence of 1.3-12.5\% and a 30-day mortality rate of up to $70 \%(3,4)$. A physical examination, ultrasonography and imaging are promising means for the diagnosis of AAA (2); to date, surgical intervention remains the only effective treatment method for AAA (5). However, any possible pharmacological target for AAA therapy remains unavailable (6). In this context, novel therapeutic strategies for AAA are urgently required.

As a prevalent neuroprotectant applied in anesthetic surgeries, dexmedetomidine (Dex) is able to suppress the activities of neuroendocrine hormones and inflammatory mediators (7). In addition, Dex helps to maintain a balanced and steady myocardial function and coronary blood flow (8). The administration of Dex during aortic vascular treatment can protect patients by reducing the incidence of myocardial ischemia as it effectively suppresses alterations in blood pressure and heart rate (9). According to a recent study, microRNA (miRNA/miR)-340 expression was markedly increased by Dex administration and in turn, the protective effects of Dex on the nervous system with inflammatory responses were improved, indicating the functional system of Dex and miRNAs (10). As small sequences in non-coding RNAs, miRNAs modulate various cell activities by degrading or suppressing specific mRNA translation, which connects them to many diseases including cardiovascular diseases (5). In addition, miRNAs function as promising biomarkers in relieving inflammatory diseases by controlling macrophage polarization, which is closely connected to severe inflammatory responses (11). miR-21 is a pivotal participator in negatively controlling inflammatory responses, particularly in macrophages (12). miR-21 is importantly involved in processes, such as extracellular matrix remodeling, lipid accumulation and inflammatory responses in 
cerebral aneurysms (13). A previous review article outlined the known effects of relevant miRNAs, including miR-21, in the development of AAA (14). miR-21 blocks the development of AAA and nicotine-augmented expansion (15). Moreover, it has been demonstrated that programmed cell death 4 (PDCD4) is a target gene of miR-21. PDCD4 is an inflammation- and apoptosis-related gene, and PDCD4 deficiency has been shown to ameliorate left ventricular remodeling and insulin resistance in a rat model of type 2 diabetic cardiomyopathy (16). miR-21 confers resistance against CVB3-induced myocarditis by inhibiting PDCD4-mediated apoptosis (17). Activation of SIRT1 protects against acute aortic dissection symptoms by enhancing PDCD4 signaling pathway (18). But there is no research on the role of PDCD4 in AAA, and on the combined efficacy of Dex and the miR-21/PDCD4 pathway on AAA. From the above, it is reasonable to hypothesize that there may be an interaction between Dex and the miR-21/PDCD4 axis in AAA. Thus, the present study conducted a series of experiments to verify this hypothesis. The present study aimed to provide theoretical guidance for the early prevention and development of AAA.

\section{Materials and methods}

Establishment of rat model of AAA. Male Sprague-Dawley rats [ $\mathrm{n}=84,8-10$ weeks old, weighing 380-450 g; State Key Laboratory of Biotherapy, Sichuan University, Chengdu, China, SYXK (Sichuan) 2016-178] were fed under a 12-h light-dark cycle and humidity-controlled clean cage at $24-26^{\circ} \mathrm{C}$. Food and water were freely accessible.

The establishment of the rat model of AAA was performed as previously described (19). As previously reported, the rats were anesthetized by inhaling isoflurane (4\% for induction and $2 \%$ for maintenance; $30 \% \mathrm{O}_{2}$ and $70 \% \mathrm{~N}_{2}$ at a flow rate of $650 \mathrm{ml} / \mathrm{min}$ ) during the surgery $(20,21)$. A midline laparotomy was conducted to expose a $10-\mathrm{mm}$ segment of the infrarenal abdominal aorta. The rats were then administered an intraluminal injection of $30 \mathrm{U}$ of porcine pancreatic elastase (135 U/mg; Sigma-Aldrich; Merck KGaA) via standard silicone tubes into the common femoral artery at the right side towards the aorta. The aorta was enveloped with gauze soaked in $0.5 \mathrm{~mol} \mathrm{CaCl}_{2}$ (Sigma-Aldrich; Merck KGaA) for $20 \mathrm{~min}$ with simultaneous elastase treatment. No rats died during the surgery. Subsequently, the abdominal cavity was rinsed using normal saline followed by suturing. The sutured rats were then placed in the cage again for raising. The health and behaviors of the rats were monitored every day. All rats survived to the end of the experiment.

The present study was approved and supervised by the Ethics Committee of The First Affiliated Hospital of Nanchang University. All animal experiments complied with the ARRIVE guidelines and were carried out in accordance with the National Institutes of Health Guide for the Care and Use of Laboratory Animals. Significant efforts were made in order to minimize both the number of animals used and their suffering.

Animal grouping. These rats were arbitrarily divided into 7 groups, with 12 rats in each group. The experiment included 3 parts. The sham-operated (sham) group, AAA group
(AAA model rats) and AAA + Dex group (Dex was intraperitoneally injected into the rats with AAA) were involved in the first part of the experiment. The Dex + control group (AAA rats were injected with Dex and intraperitoneally injected with antagomir (ant)-NC) and Dex + ant-miR-2 group (AAA rats were injected with Dex and intraperitoneally injected ant-miR-21) were included in the second part of the experiment. The lentivirus (LV) + negative control (NC) group (AAA rats were treated with control lentivirus) and LV-PDCD4-short hairpin RNA (shRNA) group (AAA rats were injected with LV-PDCD4-shRNA recombinant lentivirus) were included in the third part of the experiment.

The target organ of the experiment was the abdominal aorta. As previously described $(22,23)$, the drug was administered by intraperitoneal injection. The rats with AAA received an intraperitoneal injection of $50 \mu \mathrm{g} / \mathrm{kg}$ DEX (24) from the day of AAA modeling to 13 days after the surgery. In order to inhibit the expression of miR-21 in the abdominal aorta, ant-miR-21 or ant-NC (25) were injected intraperitoneally at the dose of $65 \mathrm{mg} / \mathrm{kg} / \mathrm{day}$ on the $1 \mathrm{st}$, 2nd and 3rd day after surgery. LV-PDCD4-shRNA and its control LV-NC were used to intervene with the expression of PDCD4 in the rats with AAA by injecting $5 \times 10^{7}$ infectious lentiviral particles per rat with AAA via the tail vein, as previously described $(26,27)$. The rats in the sham and AAA groups were operated in the same manner, but normal saline was used instead of protease (28). Cholesterol-conjugated ant-miR-21 (5'-UCA ACAUCAGUCUGAUAAGCUA-3') and ant-NC (5'-CAG UACUUUUGUGUAGUACAAA-3') were obtained from GenePharma, Inc. and were resuspended in sterile phosphate-buffered saline (PBS) at $37^{\circ} \mathrm{C}$ and preserved at $-20^{\circ} \mathrm{C}$. Recombinant lentivirus LV-NC and LV-PDCD4-shRNA were synthesized and packaged by GenePharma, Inc. LV-NC was a recombinant lentivirus containing pLKO.1 empty vector, and LV-PDCD4-shRNA was a recombinant lentivirus containing pLKO.1 plasmid transfected with PDCD4 shRNA fragment (5'-GCGGAGATGTTAGGGATTTG-3').

The maximum diameter of the renal inferior abdominal aorta on the day prior to the surgery was regarded as the baseline. That of the renal inferior abdominal aorta in the anesthetized rats was assessed using a diasonograph (Vivid7 Dimenson, Cytiva) on the 7 and 14th day after the surgery. Subsequently, the rats were injected with excessive pentobarbital sodium [800 mg/kg; (29)]. The disappearance of reflexes, respiratory arrest and cardiac arrest were observed, which indicated that euthanasia was successful. The abdominal aorta was flushed using cold PBS and transected through the left ventricle. Subsequently, the aorta, from the left renal artery to the bifurcation, was isolated from connective and fat tissues under a microscope (Leica Microsystems $\mathrm{GmbH}$ ) (30). The abdominal aorta at the perfusion segment and the abdominal aorta approximately $5 \mathrm{~mm}$ from its proximal and distal ends were selected. Samples from each group were randomly assigned into 2 groups, with 6 samples in each group. The partial samples were fixed with $4 \%$ paraformaldehyde and reserved for histological analysis and the rest were used for reverse transcription-quantitative polymerase chain reaction (RT-qPCR) and western blot analysis.

Histological analysis. The tissues already fixed with $4 \%$ paraformaldehyde were embedded in paraffin. The 5 -mm-thick 
Table I. Sequences of primers used for RT-qPCR.

\begin{tabular}{lll}
\hline Gene & \multicolumn{1}{c}{ Forward $\left(5^{\prime}-3^{\prime}\right)$} & \multicolumn{1}{c}{ Reverse $\left(5^{\prime}-3^{\prime}\right)$} \\
\hline MMP-2 & GGAATGCCATCCCTGATAACCT & CTTCACGCTCTTGAGACTTTGGT \\
MMP-9 & CTTTGTAGGGTCGGTTCT & CCTGTGAGTGGGTTGGATT \\
MCP-1 & CTATGCAGGTCTCTGTCACGCTTC & CAGCCGACTCATTGGGATCA \\
TNF- $\alpha$ & TCAGTTCCATGGCCCAGAC & GTTGTCTTTGAGATCCATGCCATT \\
IFN- $\gamma$ & GAAAGCCTAGAAAGTCTGAAGAAC & GCACCGACTCCTTTTCCGCTTCCT \\
IL-6 & ACTCACCTCTTCAGAACGAATTG & CCATCTTTGGAAGGTTCAGGTTG \\
IL-1 $\beta$ & CATGATCCGAGATGTGGAACTGGC & CTGGCTCAGCCACTCCAGC \\
PDCD4 & TTGAGCACGGAGATACGAAC & GTCCCGCAAAGGTCAGAAAG \\
miR-21 & CCCATCTATGAGGGTTACGC & TTTAATGTCACGCACGATTTC \\
U6 & TGTAACAGACACTCCATGTGG & GCTGTCAACAGTACGCTACG \\
\hline
\end{tabular}

MMP, matrix metalloproteinase; MCP, monocyte chemoattractant protein; TNF- $\alpha$, tumor necrosis factor- $\alpha$; IFN- $\gamma$, interferon- $\gamma$; IL, interleukin; PDCD4, programmed cell death protein 4; miR, microRNA.

serial cross sections of the abdominal aortas were placed on microscope slides and measured with histological analysis and immunohistochemistry. These sections were then used for hematoxylin and eosin (H\&E) staining and Elastica van Gieson (EVG) staining. Following gradient dewaxing and dehydration with conventional xylene and ethanol (both from Sinopharm Chemical Reagent Co., Ltd.), the sections were stained using H\&E staining kit (G1120, Beijing Solarbio Science \& Technology Co., Ltd.) at room temperature, stained with hematoxylin for $5 \mathrm{~min}$, then washed, differentiated for $30 \mathrm{sec}$, then soaked in tap water for $15 \mathrm{~min}$, and stained with eosin for $30 \mathrm{sec}$. The sections were then stained with Verhöeff staining solution (G1598, Beijing Solarbio Science \& Technology Co., Ltd.) for $30 \mathrm{~min}$ at room temperature, rinsed with tap water, differentiated with Verhöeff differentiation solution (G1598, Beijing Solarbio Science \& Technology Co., Ltd.) for $15 \mathrm{sec}$, then treated with $95 \%$ ethanol for $5 \mathrm{~min}$, and and counterstained with eosin for $30 \mathrm{sec}$. After dyeing, the sections were dehydrated and cleared using ethanol and xylene at room temperature, and then sealed with neutral gum (G8590; Beijing Solarbio Science \& Technology Co., Ltd.) and observed under Leica DM 750 optical microscope (Leica Microsystems GmbH).

Immunohistochemistry was applied on the sections embedded in paraffin on the 14th day with antibodies (all from Abcam), including MMP-2 (1:500, ab86607), MMP-9 (1:1,000, ab38898), nuclear factor $-\kappa \mathrm{B}(\mathrm{NF}-\kappa \mathrm{B}) \mathrm{p} 65$ (1:500, ab16502) and cluster of differentiation 68 (CD68) for macrophages (1:100, ab31630). In detail, the sections free from paraffin were cultivated with primary antibodies overnight at $4{ }^{\circ} \mathrm{C}$ and at room temperature for 10 min cultured with immunoglobulin $\mathrm{G}$ secondary antibodies (1:2,000, ab205719 and ab205718) (28,31). The sections were observed under Leica DM 750 optical microscope (Leica Microsystems GmbH).

$R T$-qPCR. TRIzol reagent (Invitrogen; Thermo Fisher Scientific, Inc.) was employed to extract total RNA from the aortic tissues at the perfusion segment and approximately $5 \mathrm{~mm}$ from the proximal and distal ends with a firm compliance to its instructions. The extracted RNA concentration was determined under a 260-nm wavelength. The total RNA was reverse transcribed into cDNA. To assess the expression of miR-21 and U6, a One StepPrimeScript miRNA cDNA Synthesis kit (Takara Biotechnology Co., Ltd.) was employed to perform reverse transcription, and the remaining RNA was reverse transcribed using a PrimeScript RT Reagent kit with genomic DNA Eraser (Takara Biotechnology Co., Ltd.). The experiment cited reverse transcribed cDNA as a standard and was conducted with a firm compliance to SYBR Premix Ex Taq II (Takara Biotechnology Co., Ltd.). This RNA was measured on a LightCycler 480 (Roche Diagnostics). The primer sequences are listed in Table I. The thermocycling conditions were as follows: Pre-denaturation at $95^{\circ} \mathrm{C}$ for $5 \mathrm{~min}$, and a total of 40 cycles of denaturation at $95^{\circ} \mathrm{C}$ for $15 \mathrm{sec}$, annealing at $58^{\circ} \mathrm{C}$ for $35 \mathrm{sec}$, and extension at $72^{\circ} \mathrm{C}$ for $30 \mathrm{sec}$. The results are displayed in arbitrary units compared with the mRNA expression of $\beta$-actin or U6. The method of $2^{-\Delta \Delta C q}$ was used for quantification (32).

Western blot analysis. A total protein extraction kit (Beyotime Institute of Biotechnology, Inc.) and Nuclear and Cytoplasmic Protein Extraction kit (Thermo Fisher Scientific, Inc.) were utilized to extract protein from the abdominal aorta at the perfusion segment and approximately $5 \mathrm{~mm}$ from the proximal and distal ends. The bicinchoninic acid protein method (Beyotime Institute of Biotechnology) was introduced to determine the protein concentration. Proteins $(30 \mu \mathrm{g})$ were then run on $10 \%$ sodium dodecyl sulfate polyacrylamide gel electrophoresis and transferred onto polyvinylidene fluoride (PVDF) membranes (Thermo Fisher Scientific, Inc.). The membranes were then cultivated in PBS with $50 \mathrm{~g} / 1$ skim milk powder at room temperature for $4 \mathrm{~h}$ and then cultured with antibodies (all from Abcam), including rabbit anti-MMP-2 $(1: 2,000$, ab92536)to detect pro MMP2 and active MMP2, MMP-9 (1:1,000, ab38898) to detect pro MMP9 and active MMP9, monocyte chemoattractant protein (MCP)-1 (1:2,000, ab25124), tumor necrosis factor (TNF)- $\alpha$ (1:1,000, ab205587), interferon (IFN) $-\gamma(1: 2,000$, ab171081), interleukin (IL)- $1 \beta$ 
(1:200, ab9722), PDCD4 (1:500, ab194522), NF-кB p65 (1:500, ab16502), histone H3 antibody (1:1,000, ab176842), glyceraldehyde-3-phosphate dehydrogenase $(1: 10,000$, ab181602) and mouse anti-IL-6 (1:400, ab9324) at $37^{\circ} \mathrm{C}$ for $1 \mathrm{~h}$. Following 3 PBS washes, the PVDF membranes were cultured with peroxidase-conjugated secondary antibody goat anti-rabbit $(1: 5,000$, ab205718) or goat anti-mouse (1:5,000, ab205719) at room temperature for $1 \mathrm{~h}$. Chemiluminescent reagent ECL (PE0010, Solarbio) was used for development for $3 \mathrm{~min}$. A chemiluminescence imager ChemiDoc (Bio-Rad Laboratories, Inc.) was used for imaging, and Image Pro Plus 6.0 software (Media Cybernetics, Inc.) was used for gray value analysis.

Dual-luciferase reporter gene assay. TargetScan (http://www.targetscan.org/vert_72/) analysis used to predict the binding site between the targeting gene and miR-21. The complementary binding sequence and mutant sequence of miR-21 and PDCD4 were amplified and cloned to pmiR-GLO luciferase vector (Promega Corporation) to establish a PDCD4 wild-type plasmid and PDCD4 mutant-type plasmid. Mimic-NC and mimic-miR-21 were transfected into 293T cells (Shanghai Institute of Biochemistry, Chinese Academic of Sciences) according to the instructions provided with Lipofectamine ${ }^{\mathrm{TM}} 2000$ (Invitrogen; Thermo Fisher Scientific, Inc.). The dual-luciferase reporter gene assay system (Promega Corporation) was used to detect the luciferase activity $48 \mathrm{~h}$ later. The ratio of firefly luciferase activity to Renilla luciferase activity was calculated to reflect the relative activity. All the experiments were performed 3 times. Mimic-miR-21 and its NC were both synthesized by GenePharma, Inc.

Statistical analysis. Statistical analysis was conducted using SPSS21.0 software (IBM Corp.). Normally distributed measurement data are expressed as the means \pm standard deviation. GraphPad Prism 8.0 (GraphPad Software, Inc.) was applied for graphing. The Kolmogorov-Smirnov test indicated whether the data were normally distributed. The non-paired t-test was used for comparisons between 2 groups. One-way analysis of variance (ANOVA) was used for comparing different groups, and Tukey's test was used as a post hoc test for these data. A P-value was attained using a two-tailed test, and $\mathrm{P}<0.05$ was considered to indicate a statistically significant difference, and $\mathrm{P}<0.01$ a highly statistically significant difference.

\section{Results}

Dex attenuates the development of AAA in rat models. It was observed that the maximum diameter of the abdominal aorta in the rats with AAA was increased significantly on the 7th day, and the maximum diameter was markedly expanded during AAA modeling 1.8-fold on the 14th day $(\mathrm{P}<0.01)$. Compared with the rats with AAA on the 7 and 14th day, the administration of Dex alleviated the expanded abdominal aortic diameter (both $\mathrm{P}<0.05$; Fig. $1 \mathrm{~A}$ and $\mathrm{B}$ ). $\mathrm{H} \& \mathrm{E}$ and EVG staining revealed a smooth outer membrane of the abdominal aorta, complete structure, dense middle layer cells and a mass of wavy and lamellar black elastic fibers in the rats from the sham group; in the rats with AAA, there was a large number of infiltrating inflammatory cells and a mass of degraded elastic fibers, and the remaining ones were heavily broken without a wavy arrangement. However, Dex ameliorated the disrupted abdominal aorta in the rats with AAA (Fig. 1C). The results from immunohistochemistry revealed that in the tunica media of the abdominal aorta of rats with AAA, there were many regions that positively expressed MMP-2 and MMP-9; these effects were suppressed by Dex (Fig. 1D). In addition, the mRNA and protein levels of MMP-2 and MMP-9 in rats with AAA were decreased when Dex was administered to the rats (all $\mathrm{P}<0.01$; Fig. 1E and F).

Dex alleviates the inflammatory response in rats with AAA. The abundant infiltration of $\mathrm{CD} 8^{+}$macrophages in the abdominal aorta was witnessed in rats with AAA, which was mitigated by the administration of Dex (Fig. 2A). In addition, the upregulated mRNA and protein levels of MCP-1 in the rats with AAA were markedly inhibited by Dex (all $\mathrm{P}<0.01$; Fig. 2B and C). Furthermore, the activated expression of the inflammatory factors, TNF- $\alpha$, IFN- $\gamma$, IL- 6 and IL-1 $\beta$, triggered by AAA were suppressed by Dex (all $\mathrm{P}<0.01$; Fig. 2B and C). Similarly, the same tendency was observed when the distribution of $\mathrm{NF}-\kappa \mathrm{B}$ p 65 and the protein levels in the abdominal aortic wall nucleus were assessed (all $\mathrm{P}<0.01$; Fig. 2D and E).

High expression of miR-21 in rats with AAA treated with Dex targets $P D C D 4$. Dex can affect the occurrence of AAA in rats; however, its mechanism of action remains unclear. In a previous study, miR, as an endogenous small noncoding RNA, was found to be a potential therapeutic target to block the development of AAA (33). miR-21 can prevent the expansion of AAA (15). In the present study, RT-qPCR verified that miR-21 was also highly expressed in the abdominal aortic wall in the rats in the AAA + Dex group $(\mathrm{P}<0.01$; Fig. $3 \mathrm{~A})$. TargetScan predicted that PDCD4 may be a target gene downstream of miR-21, which was verified by dual-luciferase reporter gene assay $(\mathrm{P}<0.01$; Fig. $3 \mathrm{~B}$ and $\mathrm{C})$. The detection of the mRNA and protein levels of PDCD4 in the rats with AAA treated with the combination of Dex and ant-21 revealed that the silencing of miR-21 evidently activated PDCD4 expression (all $\mathrm{P}<0.05$; Fig. 3D and $\mathrm{E}$ ).

Ant-miR-21 promotes the development of AAA and inflammatory responses. A combination of Dex and ant-miR-21 injected into rats with AAA inhibited miR-21 expression, thereby damaging the abdominal aortic structure and enhancing AAA expansion, which reversed the favorable results induced by Dex (all $\mathrm{P}<0.01$; Fig. $4 \mathrm{~A}-\mathrm{C}$ ), while promoting the levels of MMP-2 and MMP-9 (all P<0.01; Fig. 4D). Similarly, ant-miR-21 activated the inflammatory responses and NF- $\kappa \mathrm{B}$ p65 expression (all $\mathrm{P}<0.01$; Fig. 4E and F).

Inhibition of PDCD4 expression attenuates the development of AAA and inflammatory responses. LV-PDCD4-shRNA was utilized to limit PDCD4 expression in rats with AAA, thus attenuating AAA expansion (all $\mathrm{P}<0.01$; Fig. $5 \mathrm{~A}$ and $\mathrm{B}$ ) and reconstructing the structure and elastic fibers in the 

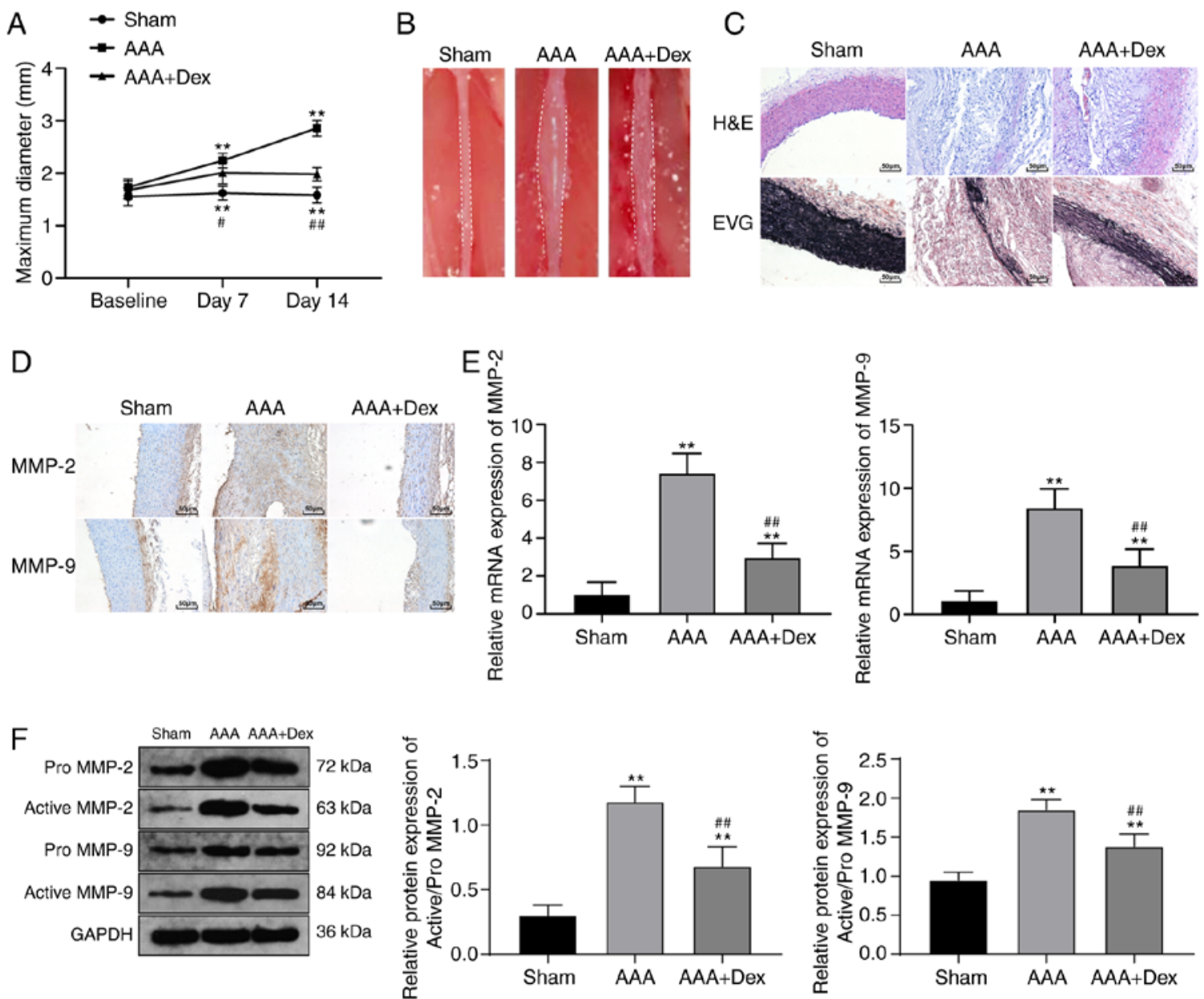

Figure 1. Dex ameliorates the development of AAA in rat models. (A) The maximum diameter of the abdominal aorta in the rats with AAA over time was detected. (B) Dex mitigated the extended abdominal aorta on the 14th day after the surgery. (C) Results from H\&E and EVG staining revealed that Dex relieved the disrupted structure of the abdominal aortic wall and the distribution of the wavy black elastic fibers in rats with AAA. (D) Immunohistochemistry was performed to verify the positively expressed regions of MMP-2 and MMP-9 in the abdominal aortic wall, and the positive expression is displayed in brown color. (E) RT-qPCR indicated the alterations in the expression of MMP-2 and MMP-9 in the abdominal aortic wall. (F) Western blot analysis revealed that Dex reduced the protein levels of MMP-2 and MMP-9 in the abdominal aortic wall of rats with AAA. Data are expressed as the means \pm standard deviation; $\mathrm{n}=6$ in each group. (A) One-way ANOVA was used to analyze the data; (E and F) one-way ANOVA was applied to evaluate the data. ${ }^{* *} \mathrm{P}<0.01$, compared with the sham group; ${ }^{\#} \mathrm{P}<0.05,{ }^{\# \#} \mathrm{P}<0.01$, compared with the AAA group. Dex, dexmedetomidine; AAA, abdominal aortic aneurysm; H\&E, hematoxylin and eosin; EVG, Elastica van Gieson; MMP, matrix metalloproteinase.

abdominal aorta (both $\mathrm{P}<0.01$; Fig. $5 \mathrm{C}$ and D). Furthermore, the levels of MMP-2 and MMP-9, as well as those of inflammatory factors were suppressed (all $\mathrm{P}<0.01$; Fig. $5 \mathrm{E}$ and $\mathrm{F}$ ). Similarly, the expression of NF- $\mathrm{BB}$ p 65 , the key protein in modulating inflammatory responses, was also downregulated and inactivated by LV-PDCD4-shRNA ( $\mathrm{P}<0.01$; Fig. 5G).

\section{Discussion}

AAA involves a series of alterations in the aortic wall structure, characterized by a thinning adventitia and media caused by extracellular matrix degradation and the insufficiency of smooth muscle cells (34). Dex is a highly selective $\alpha_{2}$-adrenoceptor agonist that is conducive in anti-inflammatory functions (35). miR-21 plays a significant role in the modulation of apoptosis and the proliferation of vascular wall smooth muscle cells in the progression of AAA, thereby protecting the progression of AAA (15). Among the theories on AAA, only a few are related to Dex; therefore, the present study aimed to explore novel therapies for AAA based on
Dex. Consequently, the findings presented herein revealed that Dex mitigated AAA by regulating the miR-21/PDCD4 signaling pathway.

First, decreased sizes of the abdominal aorta in the rats treated with Dex were observed; the decreased expression of MMP-2 and MMP-9 was also observed. Increasing data have confirmed that MMPs may be a potential treatment in inflammatory diseases (36). Among the growing MMPs in the aortic wall, MMP-2 and MMP-9 are notably increased in AAA (37). The increased activity of MMPs can promote the degradation of elastin and collagen in the arterial wall, resulting in the expansion of the arterial wall, and the inflammation in the aneurysm wall will also destroy the extracellular matrix. These mechanisms promote the formation of AAA (38). Dex has been found to be able to promote alterations in MMPs to exert anti-inflammatory effects (39). Of note, the present study found that Dex reduced the expression of MCP- 1 and that of the inflammatory factors, TNF- $\alpha$, IFN- $\gamma$, IL- 6 and IL- $1 \beta$, in the rats with AAA. MCP- 1 is involved in T-lymphocyte differentiation and chemokines 
A
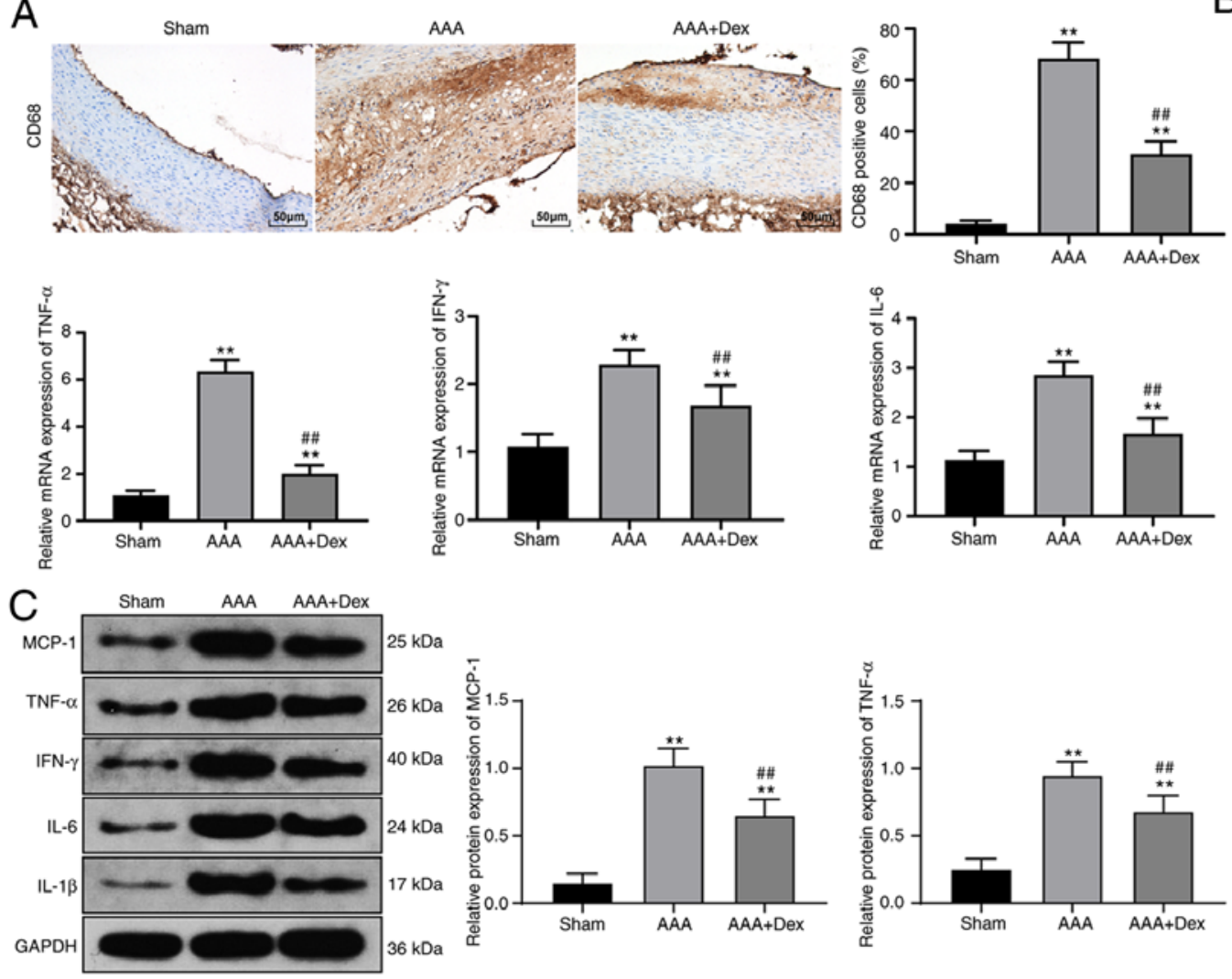

$\mathrm{B}$
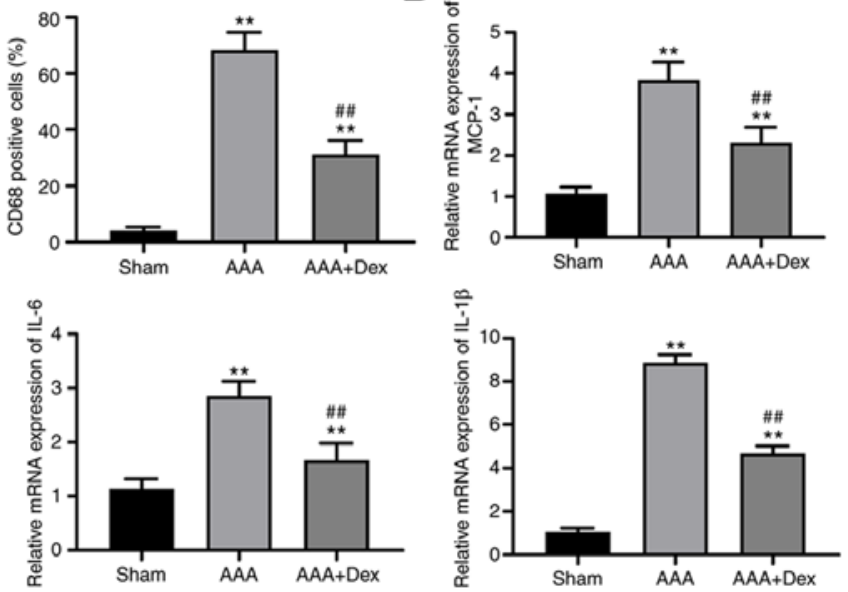
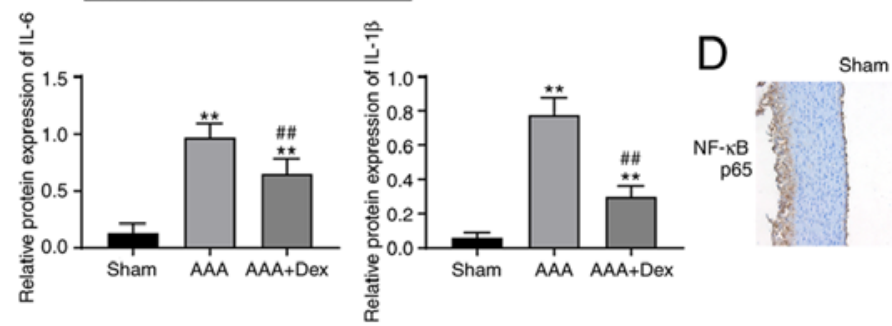

$\mathrm{E}$

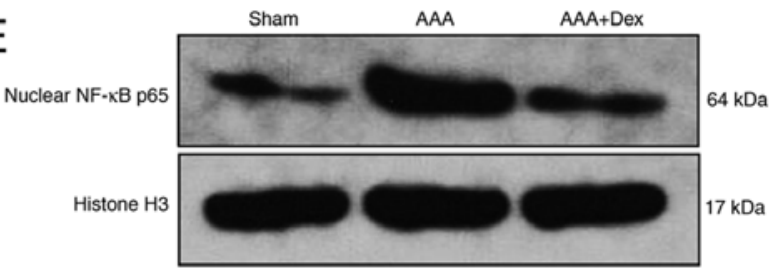

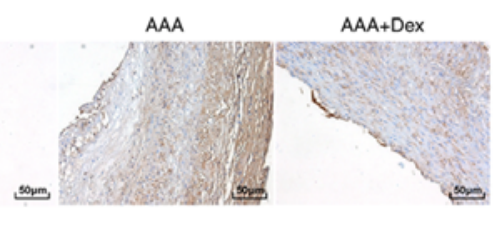
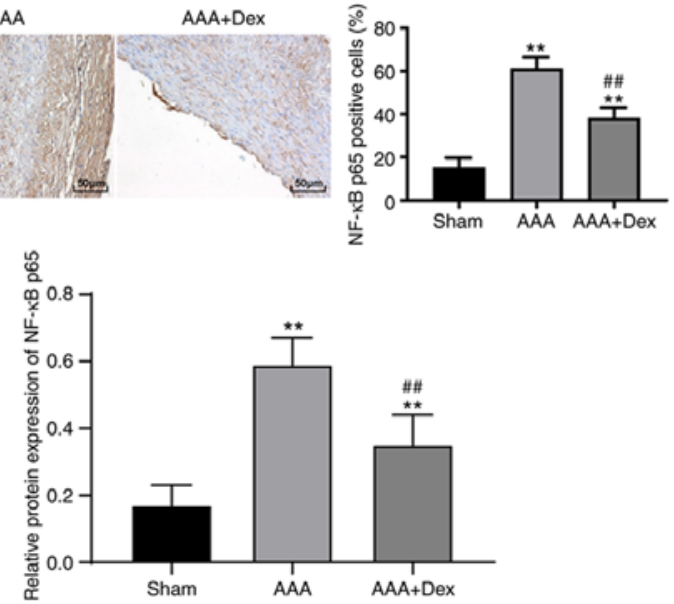

Figure 2. Dex alleviates the inflammatory reaction in rats with AAA. (A) Immunohistochemistry suggested that Dex mitigated the infiltrating CD68 ${ }^{+}$macrophages in the abdominal aorta, and the macrophage-infiltrated part is displayed in brown color. (B and C) Upregulated levels of MCP-1, TNF- $\alpha$, IFN- $\gamma$, IL-6 and IL-1 $\beta$ in the abdominal aortic wall were decreased following the administration of Dex, as shown by the results of RT-qPCR and western blot analysis. (D) Upregulated expression of NF- $\mathrm{KB}$ p65 in the abdominal aortic wall nucleus was decreased after the addition of Dex, as shown by immunohistochemistry, and the positive expression is displayed in brown color. (E) Upregulated expression of NF-кB p65 in the abdominal aortic wall nucleus was decreased following the addition of Dex, as shown by western blot analysis. Data are expressed as the means \pm standard deviation; $\mathrm{n}=6$ in each group. One-way ANOVA was applied to determine the statistical significance of the data. ${ }^{*} \mathrm{P}<0.01$, compared with the sham group; ${ }^{\# \#} \mathrm{P}<0.01$, compared with the AAA group. Dex, dexmedetomidine; AAA, abdominal aortic aneurysm; MCP-1, monocyte chemoattractant protein 1 ; TNF- $\alpha$, tumor necrosis factor- $\alpha$; IFN- $\gamma$, interferon $\gamma$; IL, interleukin.

controlling monocyte chemotaxis, which are involved in the pathological progression of atherosclerosis and inflammatory diseases (40). Dex has been shown to exert suppressive effects on MCP-1 in astrocytes via the $\alpha_{2}$-adrenoceptor (41). According to a previous study using mice with myocardial ischemia/reperfusion, following Dex treatment, the TNF- $\alpha$, IL-6 and IL-1 $\beta$ levels were all decreased (42). Thus, Dex may be helpful in palliating AAA.
In the present study, miR-21 was found to be strongly expressed in rats with AAA treated with Dex. The expression of miR-21 related to the endothelium has been shown to be markedly increased in atherosclerotic AAA tissues (43). In another study, the overexpression of miR-21 using a lentivirus promoted aortic aneurysm cell growth and suppressed cell apoptosis, preventing the progression of AAA (15). The results of the present study also verified that the addition 
A
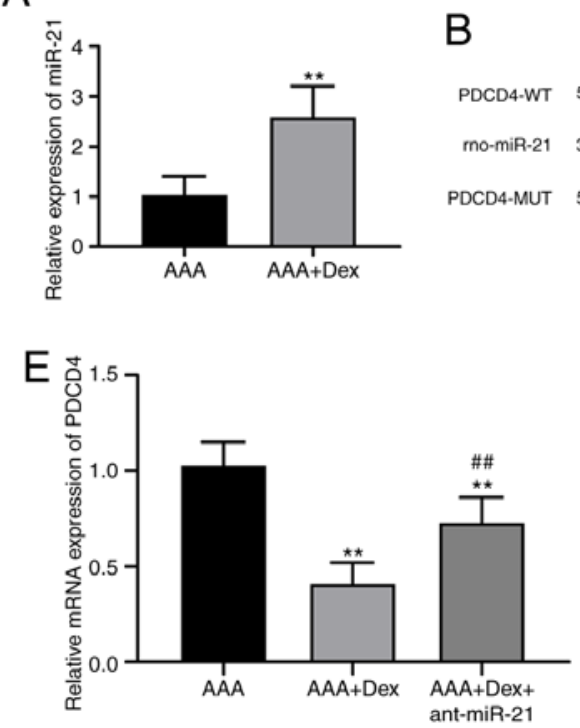

C

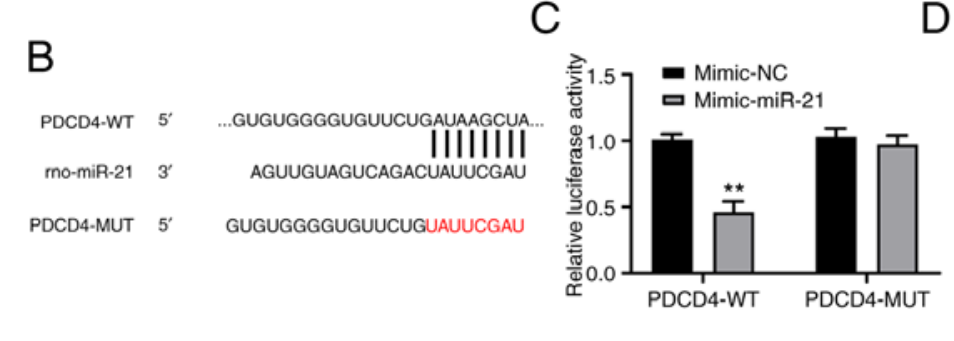

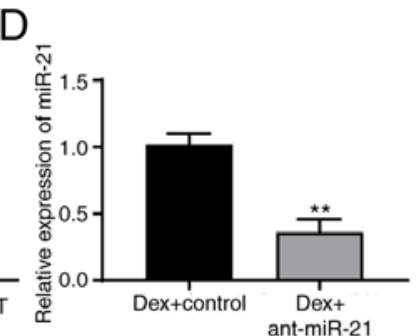

F
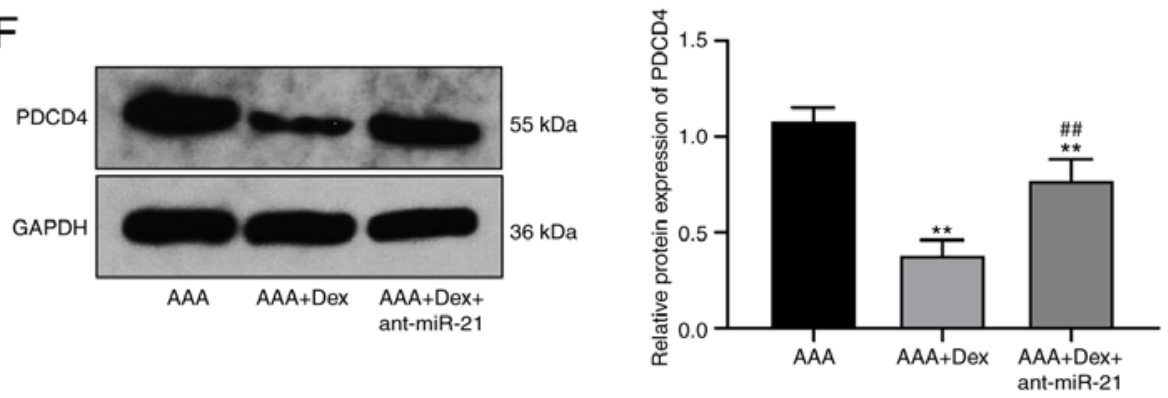

Figure 3. High expression of miR-21 in rats with AAA treated with Dex binds to PDCD4. (A) RT-qPCR revealed that miR-21 was highly expressed in the abdominal aorta. ${ }^{* *} \mathrm{P}<0.01$, compared with the AAA group. (B) The target binding sites between miR-21 and PDCD4 were predicted using TargetScan. (C) The target binding relationship between miR-21 and PDCD4 was verified by a dual-luciferase reporter gene assay. (D) RT-qPCR detected miR-21 expression following antagomiR-21 treatment. (E and F) RT-qPCR and western blot analysis suggested that suppressed PDCD4 mRNA expression in rats with AAA was reversed with the silencing of miR-21. Data are expressed as the means \pm standard deviation; $n=6$ in each group. (A and D) The t-test was used for analyzing the comparisons. (C) One-way ANOVA was applied to evaluate the data. ${ }^{* *} \mathrm{P}<0.01$, compared with the mimic group. (E and F) One-way ANOVA was applied to evaluate the data. ${ }^{* *} \mathrm{P}<0.01$, compared with the AAA group; ${ }^{\#} \mathrm{P}<0.01$, compared with the AAA + Dex group. Dex, dexmedetomidine; AAA, abdominal aortic aneurysm; PDCD4, programmed cell death protein 4.

of ant-miR-21 aggravated AAA expansion and inflammatory responses and activated $\mathrm{NF}-\kappa \mathrm{B}$. As a previous study demonstrated, the silencing of miR-21 led to an evident growth of inflammatory factors, such as TNF- $\alpha$, IL- 6 and IL-1 $\beta$ in cardiac cells and tissues of mice with myocardial infarction (44). NF- $\kappa \mathrm{B}$ represents a major activator of immune homeostasis and inflammation as it regulates various significant anti- or pro-inflammatory factors in a number of inflammatory diseases, including autoimmune disease, atherosclerosis, inflammatory bowel disease and even certain types of cancer with inflammatory components (45). A previous study demonstrated that following the attenuation of AAA with downregulated inflammatory responses, NF- $\kappa \mathrm{B}$ was markedly inhibited (46). Similarly, the silencing of miR-21 has been shown to aggravate NF- $\kappa \mathrm{B}$ expression in peritonitis (47), indicating a negative association between miR-21 and $\mathrm{NF}-\kappa \mathrm{B}$ in inflammatory diseases. Additionally, it was noted that miR-21 targeted PDCD4. The highly expression of PDCD4 serves as an engine of mitochondrial function loss and cardiomyocyte apoptosis caused by hypoxic injury (48). According to a recent study on cardiovascular diseases, PDCD4 was the direct target of miR-21 in vascular smooth muscle cells (49), which is in accordance with the findings of the present study. In addition, the overexpression of miR-21 and silencing of PDCD4 can both contribute to a significantly decreased inflammatory response (50). Furthermore, in the present study, the inhibition of PDCD4 mitigated AAA expansion and inflammatory responses, and inactivated $\mathrm{NF}-\kappa \mathrm{B}$. It has also been shown that in macrophage foam cells, which serve as an engine in atherosclerotic malignancy, the loss of PDCD4 improves cell autophagy, thus reversing the conversion from macrophages to foam cells and alleviating atherosclerosis (51). PDCD4 augments inflammatory responses by upregulating $\mathrm{NF}-\kappa \mathrm{B}$ expression (52). Another study demonstrated that in pigs with coronary microembolization, the PDCD4, NF- $\kappa \mathrm{B}$ and TNF- $\alpha$ levels were all increased (53). Furthermore, the suppression of PDCD4 was conducive to treating cardiac dysfunction resulting from coronary microembolization (54). In addition, the inactivation of $\mathrm{NF}-\kappa \mathrm{B}$ expression by Dex treatment decreased the expression of inflammatory factors, including TNF- $\alpha$, IL-6 and IL-1 $\beta$ (55). Thus, the downregulation of the miR-21/PDCD4 axis relieved AAA progression.

In conclusion, the present study demonstrated that Dex attenuated AAA by overexpressing miR-21 and targeting PDCD4. These results suggest a novel approach for the treatment of AAA. In the future, the authors aim to further explore the underlying mechanisms of other targets of Dex. Further attention also needs to be paid to identifying reliable therapeutic targets for AAA. Nevertheless, the present study was a preclinical study; although the findings presented herein provide therapeutic implications for the treatment of AAA, the experimental results and effective application in clinical practice require further validation. In the present study, a model of AAA was established in rats by elastin perfusion combined with calcium chloride coating, which effectively simulated the degradation process of extracellular matrix elastin and the inflammatory reaction of the arterial wall during the formation of human AAA. The therapeutic effects of Dex and downstream miR-21 on the development of AAA in rats were investigated and a good experimental basis was established for the further study of Dex as a preventive measure for AAA. Although the establishment of a model of AAA reflects the 

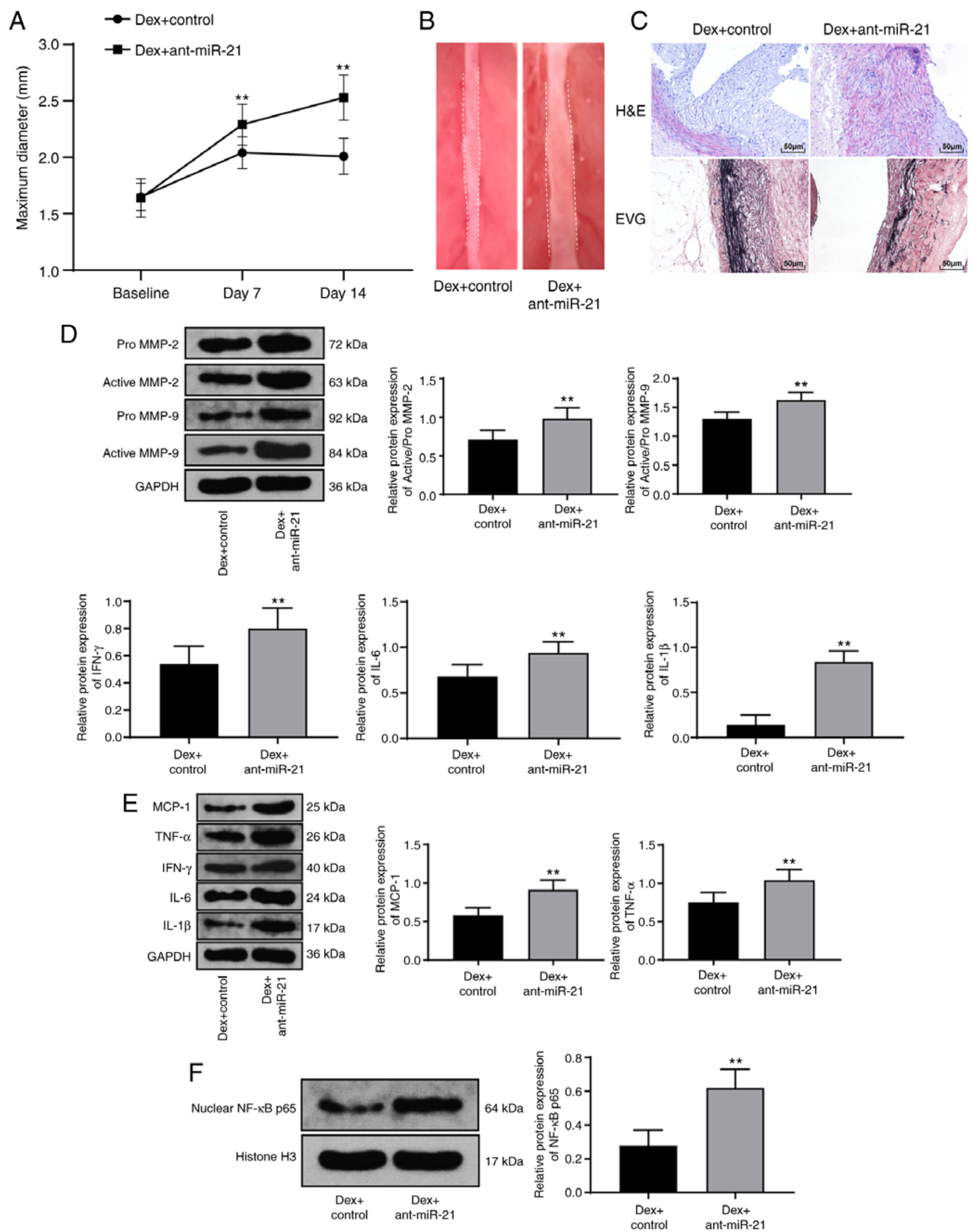

Figure 4. AntagomiR-21 enhances the development of AAA and inflammatory reactions. (A) The maximum diameter of the abdominal aorta in rats with AAA varied over time. (B) The abdominal aortic structure in rats with AAA was disrupted on the 14th day after the surgery. (C) H\&E and EVG staining revealed that AAA deteriorated after the combination of Dex and antagomiR-21 was injected. (D) Western blot analysis revealed that the levels of MMP-2 and MMP-9 were increased. (E) Western blot analysis indicated that the levels of MCP-1, TNF- $\alpha$, IFN- $\gamma$, IL-6 and IL-1 $\beta$ were increased. (F) Western blot analysis suggested that NF- $\mathrm{kB}$ p65 expression was increased. Data are expressed as the mean \pm standard deviation; $\mathrm{n}=6$ in each group. (D-F) Data were analyzed using the $\mathrm{t}$-test. (A) Data were analyzed by one-way ANOVA. ${ }^{* *} \mathrm{P}<0.01$, compared with the Dex + control group. Dex, dexmedetomidine; AAA, abdominal aortic aneurysm; $\mathrm{H} \& \mathrm{E}$, hematoxylin and eosin; EVG, Elastica van Gieson; MMP, matrix metalloproteinase; MCP-1, monocyte chemoattractant protein 1; TNF- $\alpha$, tumor necrosis factor $\alpha$; IFN- $\gamma$, interferon $\gamma$; IL, interleukin. 

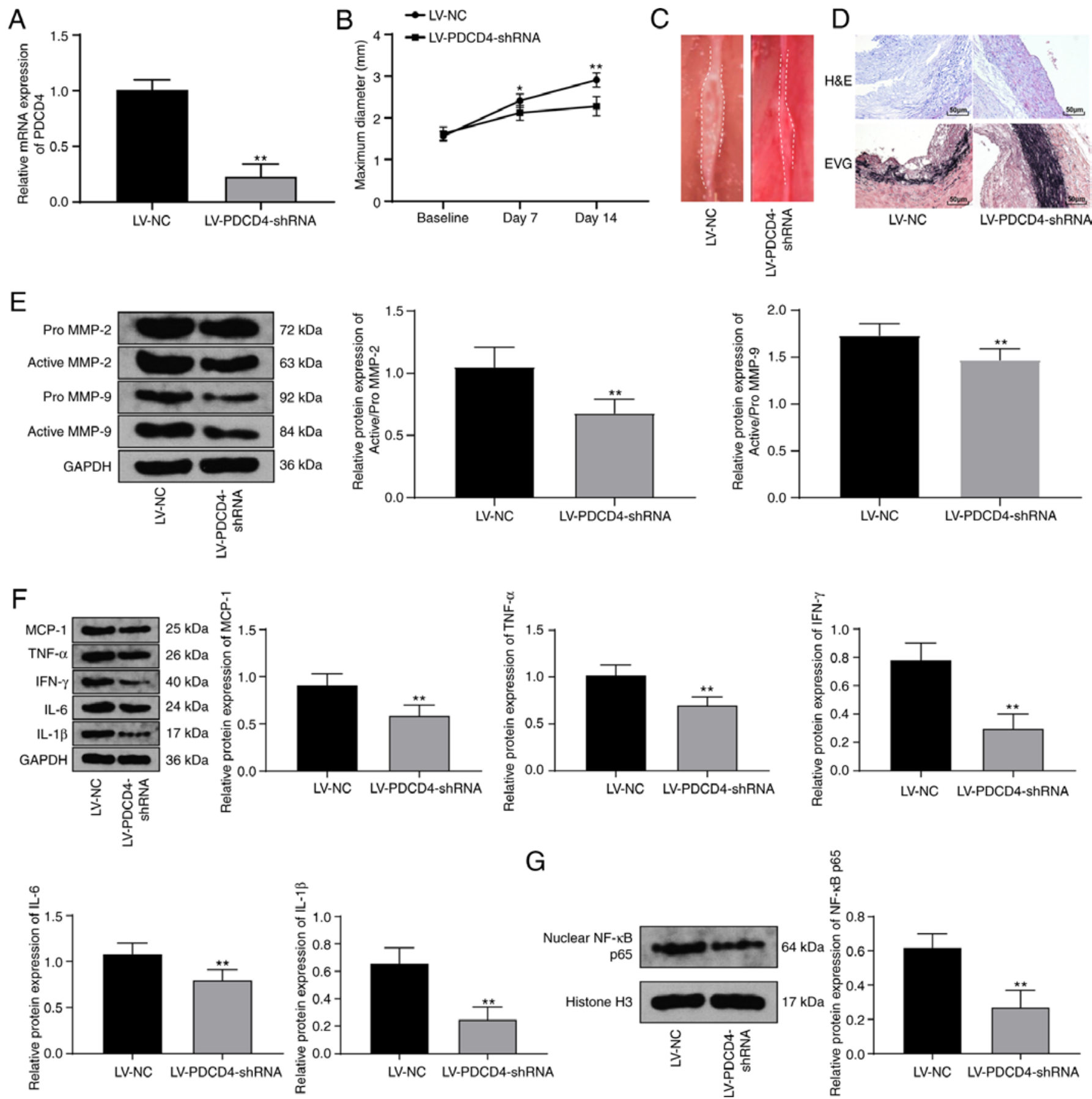

Figure 5. LV-PDCD4-shRNA relieves AAA development and inflammatory reactions. (A) RT-qPCR revealed PDCD4 mRNA expression in AAA rats following LV-PDCD4-shRNA treatment. (B) The maximum diameter of the abdominal aorta in rats with AAA varied over time. (C) Abdominal aortic structure in rats with AAA recovered on the 14th day after the surgery. (D) The structure and elastic fibers in the abdominal aorta were improved, as shown by H\&E and EVG staining. (E) Western blot analysis indicated decreased levels of MMP-2 and MMP-9. (F) Western blot analysis suggested that the levels of MCP-1, TNF- $\alpha$, IFN- $\gamma$, IL- 6 and IL-1 $\beta$ were decreased. (G) Western blot analysis revealed that NF- $\mathrm{kB}$ p65 was downregulated and inactivated. Data are expressed as the means \pm standard deviation; $\mathrm{n}=6$ in each group. (A, E, F and G) Data were analyzed using the t-test. (B) Data were analyzed by one-way ANOVA. "P<0.05, ${ }^{* *} \mathrm{P}<0.01$, compared with the LV-NC group. Dex, dexmedetomidine; AAA, abdominal aortic aneurysm; H\&E, hematoxylin and eosin; EVG, Elastica van Gieson; MMP, matrix metalloproteinase; MCP-1, monocyte chemoattractant protein 1; TNF- $\alpha$, tumor necrosis factor- $\alpha$; IFN- $\gamma$, interferon $\gamma$; IL, interleukin.

role and mechanism of Dex in the prevention of AAA to a certain extent, the cells targeted by Dex were not further explored in the present study. In addition, there are a number of factors that induce AAA clinically, and there are some differences between patients with AAA and rat models of AAA. Therefore, Dex needs to be applied in clinical practice for in-depth study. Due to the limitations of the experimental conditions and funding, the present study only discussed the effects of miR-21 and PDCD4 on the occurrence of AAA, but failed to further examine the localization of its role. In the future, the authors aim to mainly focus on the study of abdominal aorta-related cells, and the localization of miR-21 and PDCD4 in the abdominal aorta will be shown in future studies. 


\section{Acknowledgements}

Not applicable.

\section{Funding}

No funding was received.

\section{Availability of data and materials}

The data that support the findings of this study are included in this published article or are available from the corresponding author upon reasonable request.

\section{Authors' contributions}

All the authors are the guarantors of integrity of the entire study. QY and MD contributed to the conception and design of the study. XY and JD contributed to the definition of the intellectual content and the literature research for the study. QLi and YZ contributed to performing the experiments. QLiu and RH contributed to data acquisition and analysis. QY contributed to the preparation of the manuscript. MD contributed to the manuscript review. All authors read and approved the final manuscript.

\section{Ethics approval and consent to participate}

The present study was approved and supervised by the Ethics Committee of The First Affiliated Hospital of Nanchang University. All animal experiments complied with the ARRIVE guidelines and were carried out in accordance with the National Institutes of Health Guide for the Care and Use of Laboratory Animals. Significant efforts were made in order to minimize both the number of animals used and their suffering.

\section{Patient consent for publication}

Not applicable.

\section{Competing interests}

The authors declare that they have no competing interests.

\section{References}

1. Li H, Bai S, Ao Q, Wang X, Tian X, Li X, Tong H, Hou W and Fan J: Modulation of immune-inflammatory responses in abdominal aortic aneurysm: Emerging molecular targets. J Immunol Res 2018: 7213760, 2018.

2. Keisler B and Carter C: Abdominal aortic aneurysm. Am Fam Physician 91: 538-543, 2015.

3. Altobelli E, Rapacchietta L, Profeta VF and Fagnano R: Risk factors for abdominal aortic aneurysm in population-based studies: A systematic review and meta-analysis. Int J Environ Res Public Health 15: 2805, 2018.

4. Tchana-Sato V, Sakalihasan N and Defraigne JO: Ruptured abdominal aortic aneurysm. Rev Med Liege 73: 296-299, 2018 (In French).

5. Joviliano EE, Ribeiro MS and Tenorio EJR: MicroRNAs and current concepts on the pathogenesis of abdominal aortic aneurysm. Braz J Cardiovasc Surg 32: 215-224, 2017.

6. Wang YD, Liu ZJ, Ren J and Xiang MX: Pharmacological therapy of abdominal aortic aneurysm: An update. Curr Vasc Pharmacol 16: 114-124, 2018.
7. Jiang L, Hu M, Lu Y, Cao Y, Chang Y and Dai Z: The protective effects of dexmedetomidine on ischemic brain injury: A meta-analysis. J Clin Anesth 40: 25-32, 2017.

8. Zhou SZ, Li ZM, Liu XR, Zhou J, Tan XQ, Yang Y and Wei JC: Bidirectional regulatory effects of dexmedetomidine on porcine coronary tone in vitro. Med Sci Monit 23: 1621-1626, 2017.

9. Soliman R and Zohry G: The myocardial protective effect of dexmedetomidine in high-risk patients undergoing aortic vascular surgery. Ann Card Anaesth 19: 606-613, 2016.

10. Bao Y, Zhu Y, He G, Ni H, Liu C, Ma L, Zhang L and Shi D: Dexmedetomidine attenuates neuroinflammation in LPS-stimulated BV2 microglia cells through upregulation of miR-340. Drug Des Devel Ther 13: 3465-3475, 2019.

11. Essandoh K, Li Y, Huo J and Fan GC: MiRNA-Mediated macrophage polarization and its potential role in the regulation of inflammatory response. Shock 46: 122-131, 2016.

12. Sheedy FJ: Turning 21: Induction of miR-21 as a key switch in the inflammatory response. Front Immunol 6: 19, 2015.

13. Bekelis K, Kerley-Hamilton JS, Teegarden A, Tomlinson CR, Kuintzle R, Simmons N, Singer RJ, Roberts DW, Kellis M and Hendrix DA: MicroRNA and gene expression changes in unruptured human cerebral aneurysms. J Neurosurg 125: 1390-1399, 2016.

14. Adam M, Raaz U, Spin JM and Tsao PS: MicroRNAs in abdominal aortic aneurysm. Curr Vasc Pharmacol 13: 280-290, 2015.

15. Maegdefessel L, Azuma J, Toh R, Deng A, Merk DR, Raiesdana A, Leeper NJ, Raaz U, Schoelmerich AM, McConnell MV, et al: MicroRNA-21 blocks abdominal aortic aneurysm development and nicotine-augmented expansion. Sci Transl Med 4: 122ra122, 2012.

16. Zhang J, Zhang M, Yang Z, Huang S, Wu X, Cao L, Wang X, Li Q, Li N and Gao F: PDCD4 deficiency ameliorates left ventricular remodeling and insulin resistance in a rat model of type 2 diabetic cardiomyopathy. BMJ Open Diabetes Res Care 8: e001081, 2020.

17. He J, Yue Y, Dong C and Xiong S: MiR-21 confers resistance against CVB3-induced myocarditis by inhibiting PDCD4-mediated apoptosis. Clin Invest Med 36: E103-E111, 2013.

18. Zhang K, Pan X,Zheng J,Liu Y and Sun L: SIRT1 protects against aortic dissection by regulating AP-1/decorin signaling-mediated PDCD4 activation. Mol Biol Rep 47: 2149-2159, 2020.

19. Tanaka A, Hasegawa T, Chen Z, Okita Y and Okada K: A novel rat model of abdominal aortic aneurysm using a combination of intraluminal elastase infusion and extraluminal calcium chloride exposure. J Vasc Surg 50: 1423-1432, 2009.

20. Du C, Hu R, Csernansky CA, Hsu CY and Choi DW: Very delayed infarction after mild focal cerebral ischemia: A role for apoptosis? J Cereb Blood Flow Metab 16: 195-201, 1996.

21. Jeon AR and Kim JE: PDI knockdown inhibits seizure activity in acute seizure and chronic epilepsy rat models via S-nitrosylation-independent thiolation on NMDA receptor. Front Cell Neurosci 12: 438, 2018.

22. Lai CH, Chang JY, Wang KC, Lee FT, Wu HL and Cheng TL: Pharmacological inhibition of cathepsin S suppresses abdominal aortic aneurysm in mice. Eur J Vasc Endovasc Surg 59: 990-999, 2020.

23. Zhang Y, Yuan H, Bu P, Shen YH, Liu T, Song S and Hou X: Recombinant leptin attenuates abdominal aortic aneurysm formation in angiotensin II-infused apolipoprotein E-deficient mice. Biochem Biophys Res Commun 503: 1450-1456, 2018.

24. Chi X, Wei X, Gao W, Guan J, Yu X, Wang Y, Li X and Cai J: Dexmedetomidine ameliorates acute lung injury following orthotopic autologous liver transplantation in rats probably by inhibiting toll-like receptor 4-nuclear factor kappa B signaling. J Transl Med 13: 190, 2015.

25. Zampetaki A, Attia R, Mayr U, Gomes RS, Phinikaridou A, Yin X, Langley SR, Willeit P, Lu R, Fanshawe B, et al: Role of miR-195 in aortic aneurysmal disease. Circ Res 115: 857-866, 2014.

26. Li P, Yin YL, Guo T, Sun XY, Ma H, Zhu ML, Zhao FR, Xu P, Chen Y, Wan GR, et al: Inhibition of aberrant microRNA-133a expression in endothelial cells by statin prevents endothelial dysfunction by targeting GTP cyclohydrolase 1 in vivo. Circulation 134: 1752-1765, 2016.

27. Wu YL, Peng XE, Zhu YB, Yan XL, Chen WN and Lin X: Hepatitis B virus $X$ protein induces hepatic steatosis by enhancing the expression of liver fatty acid binding protein. J Virol 90: 1729-1740, 2016. 
28. Setozaki S, Minakata K, Masumoto H, Hirao S, Yamazaki K, Kuwahara K, Ikeda T and Sakata R: Prevention of abdominal aortic aneurysm progression by oral administration of green tea polyphenol in a rat model. J Vasc Surg 65: 1803-1812, 2017.

29. Zatroch KK, Knight CG, Reimer JN and Pang DS: Refinement of intraperitoneal injection of sodium pentobarbital for euthanasia in laboratory rats (Rattus norvegicus). BMC Vet Res 13: 60, 2017

30. Maegdefessel L, Spin JM, Raaz U, Eken SM, Toh R, Azuma J, Adam M, Nakagami F, Heymann HM, Chernogubova E, et al: miR-24 limits aortic vascular inflammation and murine abdominal aneurysm development. Nat Commun 5: 5214, 2014.

31. Kurobe H, Matsuoka Y, Hirata Y, Sugasawa N, Maxfield MW, Sata $M$ and Kitagawa T: Azelnidipine suppresses the progression of aortic aneurysm in wild mice model through anti-inflammatory effects. J Thorac Cardiovasc Surg 146: 1501-1508, 2013

32. Livak KJ and Schmittgen TD: Analysis of relative gene expression data using real-time quantitative PCR and the 2(-Delta Delta C(T)) method. Methods 25: 402-408, 2001.

33. Stather PW, Sylvius N, Sidloff DA, Dattani N, Verissimo A, Wild JB, Butt HZ, Choke E, Sayers RD and Bown MJ: Identification of microRNAs associated with abdominal aortic aneurysms and peripheral arterial disease. Br J Surg 102: 755-766, 2015.

34. Sakalihasan N, Michel JB, Katsargyris A, Kuivaniemi $\mathrm{H}$, Defraigne JO, Nchimi A, Powell JT, Yoshimura K and Hultgren R: Abdominal aortic aneurysms. Nat Rev Dis Primers 4: 34, 2018.

35. Li J, Wang H, Dong B, Ma J and Wu X: Adding dexmedetomidine to ropivacaine for femoral nerve block inhibits local inflammatory response. Minerva Anestesiol 83: 590-597, 2017.

36. Vandenbroucke RE and Libert C: Is there new hope for therapeutic matrix metalloproteinase inhibition? Nat Rev Drug Discov 13: 904-927, 2014.

37. Rabkin SW: The role matrix metalloproteinases in the production of aortic aneurysm. Prog Mol Biol Transl Sci 147: 239-265, 2017.

38. Duellman T, Warren CL, Peissig P, Wynn M and Yang J: Matrix metalloproteinase-9 genotype as a potential genetic marker for abdominal aortic aneurysm. Circ Cardiovasc Genet 5: 529-537, 2012.

39. Li J, Chen Q, He X, Alam A, Ning J, Yi B, Lu K and Gu J: Dexmedetomidine attenuates lung apoptosis induced by renal ischemia-reperfusion injury through $\alpha_{2} \mathrm{AR} / \mathrm{PI} 3 \mathrm{~K} / \mathrm{akt}$ pathway. J Transl Med 16: 78, 2018.

40. Bianconi V, Sahebkar A, Atkin SL and Pirro M: The regulation and importance of monocyte chemoattractant protein-1. Curr Opin Hematol 25: 44-51, 2018

41. Liu H, Davis JR, Wu ZL and Abdelgawad AF: Dexmedetomidine attenuates lipopolysaccharide induced MCP-1 expression in primary astrocyte. Biomed Res Int 2017: 6352159, 2017.

42. Sun Y, Jiang C, Jiang J and Qiu L: Dexmedetomidine protects mice against myocardium ischaemic/reperfusion injury by activating an AMPK/PI3K/Akt/eNOS pathway. Clin Exp Pharmacol Physiol 44: 946-953, 2017.

43. Kin K, Miyagawa S, Fukushima S, Shirakawa Y, Torikai K, Shimamura K, Daimon T, Kawahara Y, Kuratani T and Sawa Y: Tissue- and plasma-specific MicroRNA signatures for atherosclerotic abdominal aortic aneurysm. J Am Heart Assoc 1: $\mathrm{e} 000745,2012$.
44. Yang L, Wang B, Zhou Q, Wang Y, Liu X, Liu Z and Zhan Z: MicroRNA-21 prevents excessive inflammation and cardiac dysfunction after myocardial infarction through targeting KBTBD7. Cell Death Dis 9: 769, 2018.

45. Mitchell JP and Carmody RJ: NF- $\mathrm{KB}$ and the transcriptional control of inflammation. Int Rev Cell Mol Biol 335: 41-84, 2018.

46. Liu YF, Bai YQ and Qi M: Daidzein attenuates abdominal aortic aneurysm through NF- $\kappa \mathrm{B}, \mathrm{p} 38 \mathrm{MAPK}$ and TGF- $\beta 1$ pathways. Mol Med Rep 14: 955-962, 2016.

47. Barnett RE, Conklin DJ, Ryan L, Keskey RC, Ramjee V, Sepulveda EA, Srivastava S, Bhatnagar A and Cheadle WG: Anti-Inflammatory effects of miR-21 in the macrophage response to peritonitis. J Leukoc Biol 99: 361-371, 2016.

48. Xu H, Cao H, Zhu G, Liu S and Li H: Overexpression of microRNA-145 protects against rat myocardial infarction through targeting PDCD4. Am J Transl Res 9: 5003-5011, 2017.

49. Liu K, Liu C and Zhang Z: IncRNA GAS5 acts as a ceRNA for miR-21 in suppressing PDGF-bb-induced proliferation and migration in vascular smooth muscle cells. J Cell Biochem 120: 15233-15240, 2019.

50. Zhu Y, Liu L, Hu L, Dong W, Zhang M, Liu Y and Li P: Effect of celastrus orbiculatus in inhibiting helicobacter pylori induced inflammatory response by regulating epithelial mesenchymal transition and targeting miR-21/PDCD4 signaling pathway in gastric epithelial cells. BMC Complement Altern Med 19: 91, 2019.

51. Wang L, Jiang Y, Song X, Guo C, Zhu F, Wang X, Wang Q, Shi Y, Wang J, Gao F, et al: Pdcd4 deficiency enhances macrophage lipoautophagy and attenuates foam cell formation and atherosclerosis in mice. Cell Death Dis 7: e2055, 2016.

52. Liang X, Xu Z, Yuan M, Zhang Y, Zhao B, Wang J, Zhang A and Li G: MicroRNA-16 suppresses the activation of inflammatory macrophages in atherosclerosis by targeting PDCD4. Int J Mol Med 37: 967-975, 2016.

53. Su Q, Li L, Zhao J, Sun Y and Yang H: Effects of trimetazidine on PDCD4/NF- $\kappa \mathrm{B} / \mathrm{TNF}-\alpha$ pathway in coronary microembolization. Cell Physiol Biochem 42: 753-760, 2017.

54. Su Q,Li L,Liu Y,Zhou Y, Wang J and Wen W: Ultrasound-Targeted microbubble destruction-mediated microRNA-21 transfection regulated $\mathrm{PDCD} 4 / \mathrm{NF}-\kappa \mathrm{B} / \mathrm{TNF}-\alpha$ pathway to prevent coronary microembolization-induced cardiac dysfunction. Gene Ther 22: 1000-1006, 2015

55. Chen Z, Ding T and Ma CG: Dexmedetomidine (DEX) protects against hepatic ischemia/reperfusion (I/R) injury by suppressing inflammation and oxidative stress in NLRC5 deficient mice. Biochem Biophys Res Commun 493: 1143-1150, 2017.

This work is licensed under a Creative Commons Attribution-NonCommercial-NoDerivatives 4.0 International (CC BY-NC-ND 4.0) License. 